Pathologe 2009 · 30:89

DOI 10.1007/s00292-009-1125-4

Online publiziert: 18. Februar 2009

(c) Springer Medizin Verlag 2009

\section{K.-M. Müller}

Institut für Pathologie, Berufsgenossenschaftliche Kliniken „Bergmannsheil”, Bochum

\title{
Methoden in der Pathologie
}

\section{Klinische Relevanz - CUP-Syndrom}

Vor 30 Jahren schrieb der im letzten Jahr verstorbene Begründer unserer Zeitschrift, Herr Professor Dr. Volker Becker (Erlangen), der uns 15 Jahre der Schriftleitung geschenkt hat, in seiner Einführung:

„Pathologie und Klinik steht nicht nur für eine einfache Verbindung zweier Partner, sondern bezeichnet Komplementärphänomene und bedeutet für unsere Zeitschrift ein Programm. So ist eine Aufgabe der Zeitschrift, Der Pathologe' die praktische Medizin mit dem Imperativ der wissenschaftlichen Durchdringung zu festigen."

$\mathrm{Zu}$ dieser Zeit war die Fülle neuer wissenschaftlicher Erkenntnisse in unserem Fach durch die stetige Erweiterung besonders der molekularpathologischen Untersuchungsverfahren in den darauf folgenden 30 Jahren nicht zu erahnen.

Mehr denn je sind intensive Diskussionen zwischen Pathologie und Klinik zur Übertragung der Ergebnisse der Grundlagenforschung in den klinischen Alltag notwendig. Zunehmend häufiger werden Wissenschaftler unseres Fachgebiets zu Original- und Übersichtsarbeiten in Zeitschriften der klinischen Disziplinen gebeten. Hierdurch ergeben sich Möglichkeiten zum aktuellen Dialog über neue wissenschaftliche Erkenntnisse z. B. zur Tumorbiologie als Basis erweiterter Klassifikationen, zu Aufgaben der diagnostischen Pathologie mit Identifizierung und Validierung klinisch relevanter molekularer Veränderungen bei Tumoren bis hin zur Bestimmung prädiktiver Biomarker.

Aus den im Jahre 2008 in Zeitschriften verschiedener medizinischer Fachgebiete des Springer-Verlags publizierten Beiträ- gen haben wir exemplarisch Arbeiten mit Schwerpunkten zum Methodenspektrum und zur klinischen Relevanz der mit molekularbiologischen Verfahren zu erhebenden Befunde zusammengefasst.

Einleitend werden Bedeutung, Aufgabenbereich und Verantwortung der $\mathrm{Pa}$ thologie unter Berücksichtigung neuer Methoden der Molekularpathologie als Notwendigkeit in der diagnostischen und prädiktiven Pathologie unter therapeutischen Gesichtspunkten begründet. Die Bedeutung des Dialogs mit der Klinik wird in einer Übersicht zur Methodik der Analysen von Nierenbiopsien besonders hervorgehoben. Die Möglichkeiten von Ergänzungen der Befunde mit etablierten Methoden werden durch Ergebnisse von Genexpressionsanalysen - erhoben an Proben der Europäischen Renalen cDNA-Bank in Zürich - bezüglich ihrer Bedeutung für die Therapie von Nierenerkrankungen diskutiert.

Methodenspektrum, Ergebnisanalysen und klinische Relevanz molekularpathologischer Untersuchungen durch Pathologie und Klinik werden an Tumoren von Nieren und Prostata sowie kleinzelligen Lungentumoren von klinischen Kollegen und Pathologen aus Jena, Hamburg, Heidelberg und Bremen dargestellt und kommentiert.

Der Bedeutung molekularpathologischer Untersuchungen bei Fragen zu Biologie, Pathogenese und Diagnostik beim "cancer of unknown primary“ (CUPSyndrom) widmen sich zwei Arbeiten aus Leipzig, Heidelberg und Würzburg. Dabei gehen auch Gesichtspunkte der täglichen Praxis mit Empfehlungen zum rationalen und rationellen Einsatz von Zusatzuntersuchungen für die Charakterisierung der Tumoren ein.

Von allen Autoren der Beiträge in diesem Heft wird die Bedeutung enger „Netzwerke“ zwischen Pathologie, Klinik, Biowissenschaften, Statistik und Industrie bei der Bearbeitung wissenschaftlicher Fragestellungen hervorgehoben. Bei den nur noch schwer überschaubaren Angeboten neuer Methoden der Molekularpathologie sind „Netzwerke“ auch zwischen den Instituten und Praxen unseres Fachs zur Kooperation beim Einsatz aufwendiger und kostspieliger Untersuchungsverfahren mit regelmäßigen Konsiliarkontakten und Konferenzen unerlässlich. Bei der gegenwärtigen Situation einer bisher nicht gekannten Wissensflut durch neue Methoden in der Pathologie ergeben sich Fragen der Abgrenzungen bzw. Überschneidungen des grundsätzlich Machbaren zu unbedingt Notwendigem bis zum prospektiv Bedeutsamen.

Mögen die Beiträge in diesem Heft den Lesern Anregungen und Entscheidungshilfen bei der täglichen Arbeit und für die zweifelsfrei aufregenden Zukunftsperspektiven unseres Fachs geben.

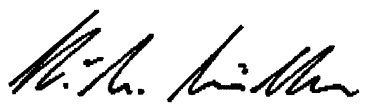

Prof. Dr. K.-M. Müller

\section{Korrespondenzadresse \\ Prof. Dr. K.-M. Müller}

Institut für Pathologie, Berufsgenossenschaftliche Kliniken "Bergmannsheil" Bürkle-de-la-Camp-Platz 1, 44789 Bochum

Klaus-Michael.mueller@rub.de 